\title{
EL CAMBIO CLIMÁTICO Y LA UTILIZACIÓN DE LAS TECNOLOGÍAS DE LA INFORMACIÓN GEOGRÁ- FICA
}

\author{
Manuel QUIRÓS HERNÁNDEZ \\ Profesor Jubilado. Departamento de Geografía \\ Universidad de Salamanca
}

Recibido: 22/01/2013

Devuelto: $04 / 07 / 2013$

Aceptado: 12/07/2013

RESUMEN: La muy diversa afectación mundial del cambio climático y el procesado de la cuantiosa cantidad de datos necesarios para su determinación hacen prácticamente imposible su estudio y análisis sin herramientas y métodos sofisticados que ayuden a globalizar resultados estadísticos que desde la escasa densidad de observatorios en muy extensas regiones del planeta son muy difíciles de interpolar, extrapolar y generalizar. Esto está llevando a una frustrante controversia entre distintas posturas científicas enfrentadas. Por eso, se hace cada vez más importante y necesaria la utilización de las TIG's que faciliten, por un lado, una mayor densidad superficial de los datos meteorológicos con el fin de analizar los impactos paramétricos del cambio climático en ámbitos locales, cuya integración permitirá definir el carácter global del fenómeno con mayor claridad; y, por otra, el manejo y computación del creciente número de datos obtenidos. Dada la profusión actual de fuentes de información del problema, se hace muy necesaria para la orientación de investigadores y sus equipos la ordenación y puesta al día de las tecnologías y métodos disponibles actualmente que pueden facilitar sus objetivos científicos. La cartografía clásica y digital para informar de resultados de las investigaciones; las plataformas aéreas y espaciales que permiten captar radiaciones terrestres indicadoras de parámetros climáticos; y los programas informáticos que faciliten los análisis estadísticos, geoestadísticos y territoriales mediante capas de datos de los distintos lugares que se computen con álgebras matriciales y permitan definiciones impensables hace muy pocos años atrás. Este artículo pretende una aproximación a estas materias.

PALABRAS CLAVE: Cambio Climático; evolución y ordenación de las TIG's, Cartografía, Teledetección, Sistemas de Información Geográfica.

\section{THE CLIMATE CHANGE AND THE USE OF GEOGRAPHIC INFORMATION TECHNOLOGY}

ABSTRACT: There are diverses impacts of global climate change, and challenges of processing the large amount of data. The determination of results makes it practically impossible both to study and analyse without tools and sophisticated methods which would eventually help to globalise statistical results. The latter due to the scant density of observatories in very vast regions of the planet make specific results very difficult to interpolate, extrapolate and generalise. All of which is leading to a frustrating controversy between confronted scien- 
tific positions. Therefore, it is becoming increasingly important and necessary the use of TIG's which, on one hand, facilitate a greater surface density of meteorological data which eventually would allow the analysis of the parametric impacts of climate change on local areas, whose integration would allow to define more clearly the global nature of this phenomenon. And on the other hand the management and computing of an increasingly accelerating number of obtained data. Given the current abundance of sources of information in relation to the issue, it has become necessarily capital for the guidance of researchers, and their research teams of management of implementation to be abreast of the technological and methodological updating which, eventually, would facilitate their scientific objectives. Both classic and digital mapping for the reporting results of investigations, as well as aerial and spatial platforms that permit to capture terrestrial radiation of climatic parameters, and those computer programs that allow the statistical analysis, geostatistical and territorial, through layers of data of the different places that counted with matrix algebras, all of them allow definitions which were merely unthinkable a few years ago.

Ultimately, this article aims to tackle and approach all these matters.

KEY WORDS: changing climate; evolution and management of the GIT's, mapping, remote sensing, geographic information systems.

\section{INTRODUCCIÓN}

Desde que en 1975 se publicó en la revista Science (American Association for the Advancement of Science) el famoso artículo de Wallace Smith Broecker (catedrático de Ciencias Ambientales y de la Tierra en la Universidad de Columbia- USA) "Climate Change: Are We on the Brink of a Pronounced Global Warming?” quedó sancionado el nombre del fenómeno global al que en el último cuarto del siglo xx y primeros decenios del presente siglo la humanidad ha dado más importancia que incluso a la preocupación por la explosión demográfica, a la que debería estar muy ligada. Broecker se preguntaba si se estaba entonces al borde de un calentamiento global pronunciado a causa de los cambios en los "conveyor belts" y termohalinas tan influyentes en los cinturones de circulación atmosférica. También se puso en valor su opinión acerca de que en el caso de que la contaminación atmosférica procedente de la actividad humana no tuviese influencia sobre el cambio climático entonces se acentuaría en los siguientes veinte años una tendencia al enfriamiento mucho mayor que la que había ocurrido en los últimos mil años y, desde luego, que la detectada desde los años cuarenta del siglo pasado. Y auguraba que en realidad en una década el enfriamiento sería ampliamente compensado por el $\mathrm{CO}_{2}$ y demás gases de efecto invernadero inyectados en la atmósfera desde los comienzos de la Revolución Industrial. Parece que muchos estudios posteriores le han dado la razón. Y que sus dos contradictorias hipótesis han abonado los campos tanto de los que propugnan el calentamiento acelerado como de los que lo niegan.

Estas afirmaciones no hicieron otra cosa que ir generalizando y difundiendo teorías e hipótesis anteriores sobre el calentamiento y el enfriamiento 
global, como las del químico y físico sueco Svante August Arrhenius que ya en 1896 predecía que el uso masivo y extendido de combustibles fósiles podría llevar a un calentamiento de la Tierra; pasando por la difusión de la famosa curva de Keeling de concentración de $\mathrm{CO}_{2}$ en la atmósfera realizada en 1958 con los datos recogidos durante más de un siglo en el observatorio de Mauna Loa en Hawai para destacar su papel en el calentamiento global, y que estudios muy recientes desvían su protagonismo a un papel fundamental en la acidificación $\left(\mathrm{H}_{2} \mathrm{CO}_{3}\right)$ de los océanos más que en su efecto invernadero atmosférico; hasta llegar a las conclusiones actuales de meteorólogos destacados, como Richard Lindzen del MIT en la II conferencia internacional sobre el cambio climático o las más definitivas del cuarto informe en 2007 del IPCC que da por muy altamente probable que el calentamiento sea causado por el aumento de gases de efecto invernadero $\left(\mathrm{CO}_{2}, \mathrm{~N}_{2} \mathrm{O}\right.$ y $\mathrm{CH}_{4}$, etc..) procedentes de la actividad humana. Sin embargo bastantes científicos, algunos prestigiosos como, por ejemplo, Bjon Lomborg (Director del Instituto medioambiental de Dinamarca), Christopher Hormer de USA, Khabibullo Abdusamatov (Observatorio Pulkovo de San Petersburgo), etc., no aceptan completamente las conclusiones de dicho cuarto informe porque creen que la concentración de $\mathrm{CO}_{2}$ ha aumentado más de un $4 \%$ en los últimos 10 años, pero el calentamiento prácticamente ha cesado en 2005 porque la cantidad de energía solar ha disminuido en el mismo periodo.

Gráfico 1. Relación entre contenido de $\mathrm{CO}_{2}$ en la atmósfera y $\mathrm{H}_{2} \mathrm{CO}_{3}$ en océanos
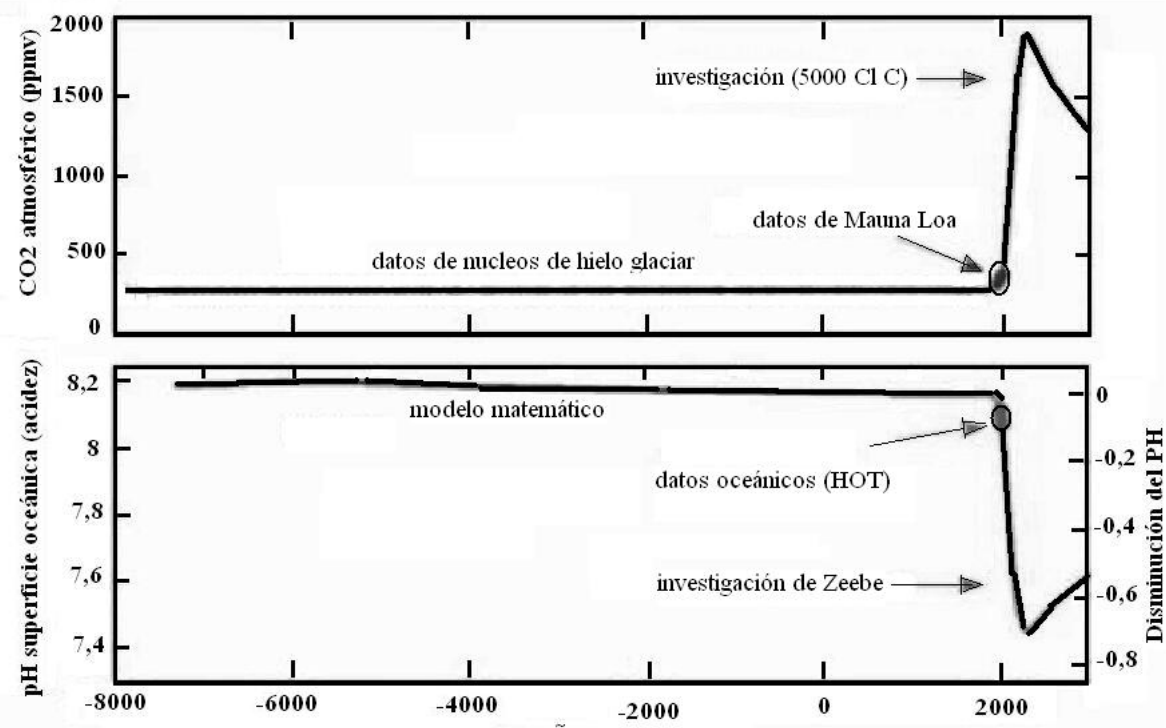

FUENTE: Adaptado de ZEEBE, R. (2012a) 
El acaparamiento de la interpretación de los informes, datos y eventos acerca del calentamiento global por parte de medios de comunicación, de políticos y de intereses económicos está llevando a deformaciones muy importantes en su percepción general, hasta llegar a trascender lo puramente geofísico y climatológico para convertirse en un fenómeno social, político y económico. Esto ha hecho que se imponga la necesidad de diferenciar claramente conceptos epistemológicos nuevos que ya son defendidos incluso por economistas de prestigio como Sachs J.D. (profesor de Economía y director del Herat Institute de la Universidad de Columbia -EE.UU- y consejero del Secretario General de las Naciones Unidas sobre las metas de desarrollo del milenio), en el sentido de diferenciar claramente la detección del cambio climático para poder determinar si un evento extremo en el plazo corto forma parte de las fluctuaciones climáticas normales o si es síntoma de un cambio más profundo y de largo plazo, y la atribución o capacidad de asignar causas probables de un suceso o fenómeno a la actividad humana o a otros factores (SACHS, 2012).

De manera, que a pesar de los grandes crecimientos en las asignaciones económicas presupuestarias que por una parte distorsionan los objetivos de cualquier proyecto de investigación para conseguir financiación pública o privada, forzándolos a conectarse con el problema del cambio climático aunque no tengan mucho que ver $\mathrm{y}$, por otra, han permitido grandes avances en la disposición de herramientas y métodos muy sofisticados que permitan ir acotando el problema hasta convertirlo en más incontrovertible, la polémica científica es posible aún y facilita argumentos en un sentido o en otro. En cualquier caso, se está produciendo un desplazamiento de la controversia desde el campo de la detección al de la atribución. Parece pues necesario un avance en los instrumentos, técnicas y métodos de investigación del problema del cambio climático, en la línea de los que ofrecen actualmente las tecnologías de la información geográfica, para impedir su manipulación extracientífica.

Actualmente se destacan en forma paradójica dos problemas para los estudiosos: En primer lugar el manejo de la enorme cantidad de datos de que se dispone. Entre otros los provinientes, por ejemplo, del SMO (Sistema Mundial de Observación), que pertenece a la OMM y es un sistema coordinado de métodos e instalaciones para observaciones meteorológicas globales sobre mar y tierra que recopila datos de más de 15 satélites; 100 boyas fondeadas, 600 a la deriva; 3.000 aeronaves; 7.300 buques; y 10.000 estaciones terrestres de sus 183 países miembros ${ }^{1}$. De otro lado, la profusión de modelos

${ }^{1}$ Una sola fuente como el sensor MODIS del satélite heliosíncrono TIERRA ofrece dos 
estadísticos y de programas informáticos para el tratamiento de los datos, que producen distintos resultados en función de la elección de los parámetros y de las variables dependientes e independientes, y provocan el que para llegar a simplificaciones y agrupaciones manejables sean elegidos en función de los conocimientos científicos que se posean, de intuiciones y de los objetivos que se pretendan.

\section{NECESIDAD PARA LA CLIMATOLOGÍA DE LAS TECNO- LOGÍAS DE LA INFORMACIÓN GEOGRÁFICA}

Es muy difícil establecer un comportamiento global de los climas sobre las bases de datos de redes de observatorios terrestres que si en unas áreas son suficientemente densas, debido a que pertenecen a países desarrollados con redes nodales urbanas consolidadas y equilibradas, en la mayoría del resto de países se produce una ausencia y/o unas condiciones técnicas deficientes en las existentes.

Conviene tener en cuenta que realmente un clima se manifiesta como una estadística de parámetros atmosféricos que tienen valores repetidos, según umbrales no muy amplios, dentro de cada estación astronómica en conjuntos extensos de la superficie terrestre; por tanto la calidad y fiabilidad de los datos, la simultaneidad estandarizada en la obtención de los mismos y el periodo de tiempo en que se han registrado son asuntos fundamentales que deberían conducir a hablar sobre todo de un cambio epistemológico-estadístico de los climas de la Tierra. Sin embargo, parece bastante clara su existencia como evento global por los síntomas que están produciendo en fenómenos tan importantes como la disminución de la banquisa ártica o la de los glaciares de montaña, así como en la percepción de las poblaciones humanas cada vez más afectadas por los meteoros extremos.

Respecto a las causas del cambio, el grupo de investigadores de referencia más importante sobre el cambio climático, agrupados por la ONU en el Panel Intergubernamental sobre el Cambio Climático (IPCC) ha ido ajustando sus informes, evolucionando desde un simple "el balance de evidencias sugiere una influencia humana discernible sobre el clima global” en el segundo informe de 1995 (SAR); pasando por las "nuevas, fuertes y evidentes pruebas" del tercer informe (TAR) de 2001; hasta llegar a que "en un $90 \%$ es muy probable que las actividades humanas hayan influido globalmente sobre el calentamiento desde 1750" del cuarto informe (AR4) de 2007. En este último además se constata un enfriamiento de la estratosfera, coincidente con

veces al día un dato de temperatura de suelo por cada cuadrado de $500 \mathrm{~m}$. de lado de la superficie terrestre, lo que supone 4.080 millones de datos térmicos diarios para todo el planeta. 
el calentamiento de $0,65^{\circ} \mathrm{C}$ de la troposfera en los anteriores 50 años; también el incremento del vapor de agua y consiguiente cambio en los patrones de precipitación y presión a pequeña escala de los modelos teóricos estadísticos, que son bastante concordantes con las observaciones empíricas, especialmente la disminución general de la presión atmosférica en los polos que redireccionan las rutas de las borrascas. Sin embargo, los modelos no atribuían demasiada influencia sobre los ciclones tropicales y además preveían un mayor incremento térmico en la troposfera que en la superficie terrestre, en contra de lo que se evidencia en las observaciones directas; además de que no concuerdan en los océanos y a pequeñas escalas sobre la superficie terrestre ni explican los eventos extremos (HEGERL, 2007). Las causas se agrupaban del siguiente modo: 1) variabilidad interna del clima o variabilidad de fondo, es decir a causa de factores propios; 2) factores externos al clima pero naturales, como los ciclos solares o las erupciones volcánicas; y 3) factores artificiales, como la inyección en la atmósfera de gases de efecto invernadero y aerosoles procedentes de la actividad humana. Es muy difícil computar las distintas atribuciones de un modo cuantitativo para diferenciar los denominados forzamientos sobre el clima y sus respuestas, debido a las incertidumbres en las observaciones y en los modelos de simulación. La detección del cambio climático se basa en $a$ ) la compresión del cambio natural mediante los paleo-registros y los modelos de simulación asociados, que en general indican que actualmente existe más $\mathrm{CO}_{2}$ que nunca y un calentamiento medio progresivo en los últimos 200 años; $b$ ) el desarrollo y la aplicación de técnicas estadísticas avanzadas para la identificación de tendencias; $c$ ) los análisis de las observaciones para comprender sus limitaciones, valorar las incertidumbres y validar los modelos matemáticos para conseguir lo que se conocen como producciones retrospectivas (wave hindcast); y d) la identificación y el diseño de índices multivariantes apropiados. El propio informe AR4 incluye las incertidumbres producidas en los datos obtenidos mediante teledetección y globos sonda (MILLER, 2009).

Otros estudios también ponen de relieve que la conclusión del llamado "forzamiento antrópico del cambio climático" depende en exceso de modelos físicos e informáticos que aún son demasiado simples. Y esto pese a los grandes esfuerzos que se realizan para hacerlos más potentes, como los del Grupo de Expertos sobre Detección e Índices del Cambio Climático (ETCCD) de la OMM que hacen que no desaparezcan problemas y grandes incertidumbres en los cálculos de retorno estadístico y destacan problemas en la interoperabilidad de los formatos digitales de los datos procedentes de distintas redes, o en sus restricciones de uso. Especialmente se destacan la escasa disponibilidad y las limitaciones en la calidad de los datos de las series históricas existentes para detectar la variabilidad, que además están muy 
poco disponibles en formatos digitales y recogidas en bases de datos bastante incompatibles entre sí. Esto hace muy necesarias: 1) la verificación de grandes errores; 2) las pruebas de tolerancia (outliers); 3) la detección de datos fuera de rango de la variabilidad esperable; 4) las pruebas de consistencia interna; 5) las pruebas de coherencia temporal; y 6) las pruebas de coherencia espacial. En estos dos últimos apartados se incluyen los cambios de instrumentos y su protección, los de localización y ubicación y las técnicas de tratamiento estadístico. Esto explica los continuados intentos de homogeneización y estandarización que existen como, por ejemplo, los de la Acción Europea Cost Home (BRUNET, 2010). Y eso que ya se han desarrollado gran cantidad de índices, como los 27 agrupados en cinco conjuntos que se basan en percentiles para umbrales relativos, índices absolutos, indicadores de umbrales absolutos, indicadores de duración y otros (ALEXANDER, 2006).

Desde los comienzos mismos de la toma en consideración del cambio climático se tiene constancia de estos problemas en las investigaciones y de la necesidad de utilizar los datos suministrados por la teledetección. Así ha habido grandes proyectos tempranos, como el ISLSCP (Internacional Satellite Land-surface Climatology Project) que realizó una Conferencia-Taller en 1992 en Columbia -Maryland- (USA) en la que se reunieron 240 científicos para revisar los modelos, métodos y algoritmos, así como la estructura y normalización de los datos obtenidos desde los satélites artificiales; marcándose como grandes objetivos: 1) la revisión de los modelos de los balances energéticos, especialmente los de tierra-atmósfera; 2) el examen del papel de la teledetección y de los algoritmos para el tratamiento conjunto de los datos de los observatorios terrestres y los de los satélites; y 3) la determinación de las futuras líneas de investigación. En este Taller-Conferencia se produjo la dedicación de las investigaciones hacia la aclaración del cambio climático, basándose en los balances de energía y radiación en las capas bajas de la atmósfera, datos de precipitación de lluvia, cubiertas de nieve e hielo, y de humedad en los suelos recogidos en el decenio 1985-1995. Los distintos trabajos o iniciativas condujeron al diseño de pruebas y experimentos: INICIATIVA I que diseñaba una resolución de celdas normalizadas de rectángulos de $1^{\circ}$ de latitud por $1^{\circ}$ de longitud para la obtención mensual de datos de hidro-meteorología (temperatura, humedad, viento, precipitación, escorrentía, hielo y nieve, balances de radiación -aumentando la escala del proyecto anterior ISCPP que estudiaba la radiación incidente fotosintéticamente activa entre 0,4 y $07 \mu \mathrm{m}$ (PAR)-, nubosidad, y homogeneización de la clasificación de usos de suelos); INICIATIVA II para producir versiones mejoradas de los conjuntos de datos de la primera iniciativa; y la INICIATIVA III que coordinaba los ejes de investigación en cuanto méto- 
dos, algoritmos y trabajos de campo y su comunicación dentro de la LSC (Land Science Community) (SELLERS, 1995).

Cuadro 1. Variables fundamentales y resoluciones propuestas para los modelos Agua-Energía-Carbono por el ISLSCP (1995)

\begin{tabular}{|c|c|c|c|c|c|c|}
\hline & \multicolumn{3}{|c|}{ Requerimientos } & \multicolumn{3}{|c|}{ Fuente / Precisión } \\
\hline & $\begin{array}{l}\text { Resolución es- } \\
\text { pacial (Km.) }\end{array}$ & $\begin{array}{l}\text { Resolución } \\
\text { temporal }\end{array}$ & Precisión & $4 D D A$ & Satélite & Otras \\
\hline Albedo & $250 \times 250$ & mensual (*) & 0,02 & & 0,03 & Clasificación \\
\hline SW surf & $250 \times 250$ & mensual $(*)$ & $10 \mathrm{~W} / \mathrm{m}^{2}$ & & $20 \mathrm{~W} / \mathrm{m}_{2}$ & \\
\hline PAR & $250 \times 250$ & mensual $(*)$ & $10 \mathrm{~W} / \mathrm{m}^{2}$ & & $20 \mathrm{~W} / \mathrm{m}_{2}$ & \\
\hline$L W$ met & $250 \times 250$ & mensual (*) & $10 \mathrm{~W} / \mathrm{m}^{2}$ & $10 \mathrm{~W} / \mathrm{m}^{2}$ & & \\
\hline Evapotranspiración & $250 \times 250$ & mensual (*) & 0,03 mm/día & $10 \mathrm{~W} / \mathrm{m}^{2}$ & & \\
\hline Precipitación & $250 \times 250$ & mensual $(*)$ & 0,03 mm/día & & $50 \%$ & $\begin{array}{l}\text { Observaciones } \\
\text { en superficie }\end{array}$ \\
\hline $\begin{array}{l}\text { Propiedades hidraúli- } \\
\text { cas del suelo }\end{array}$ & $1--10$ & una vez & & & & FAO \\
\hline Rugosidad del suelo & $250 \times 250$ & estacional & $300 \%$ & & & Clasificación \\
\hline $\begin{array}{l}\begin{array}{l}\text { Relieve y redes de } \\
\text { drenaje }\end{array} \\
\end{array}$ & 1 & una vez & $\begin{array}{c}\text { corrientes } \\
\text { consistentes }\end{array}$ & & & USGS/DoD \\
\hline $\begin{array}{l}\text { Meteorología próxima } \\
\text { al suelo }\end{array}$ & $250 \times 250$ & diario (*) & varios & & & $\begin{array}{c}\text { Observaciones } \\
\text { en superficie }\end{array}$ \\
\hline
\end{tabular}

$\left({ }^{*}\right)$ Más el ciclo diurno y la variabilidad interdiaria

Gráfico 2. Calentamiento Global. Observaciones empíricas y modelos

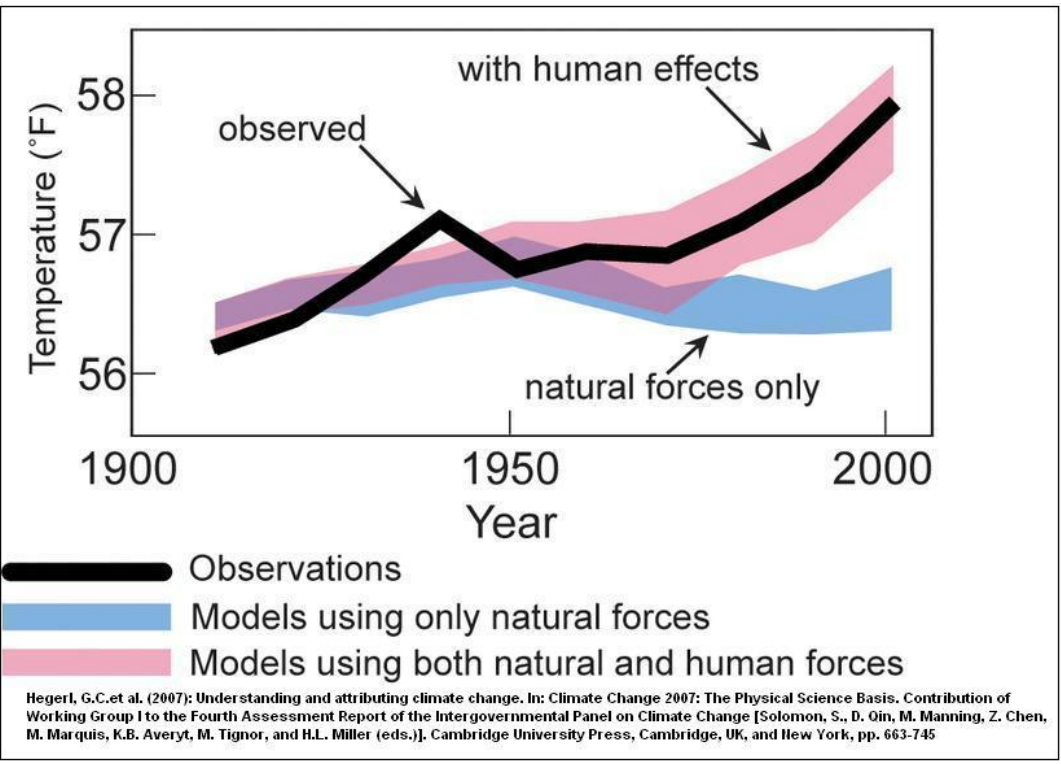

FuENTE: HEGERL, G. C. et al. (2007) 
Las mejoras de las técnicas epistemológicas y estadísticas utilizadas ayudan también a ordenar los conjuntos de datos y deducir comportamientos climatológicos en grandes y pequeños espacios terrestres. Puede servir de ejemplo el que computando datos desde 1961 hasta 2007, mediante la técnica de Componentes Principales se han detectado comportamientos diferenciales respecto al cambio climático en tres grandes subregiones climatológicas occidentales: Centro Norte (CN); Mediterránea (M) y Atlántica (A) (SÁNCHEZ LORENZO, 2012). Sólo en la evolución de los modelos, sus sistemas de computación y en las fuentes de los datos se puede esperar encontrar solución a estos problemas científicos.

Además de basarse en síntomas indirectos, como que la extensión del hielo ártico ha disminuido en los últimos 20 años de un modo coincidente con los modelos predictivos, la afirmación del cambio climático y el calentamiento global se fundamenta, por un lado, en la variación de los valores de los parámetros recogidos en los observatorios a lo largo de la era industrial, en el sentido de una elevación de las temperaturas; pero habrá que ponderar más precisamente el incremento del efecto "isla urbana de calor" al que se ven sometidos la mayoría de los entornos de los observatorios (GALLO, 1993). Por otra parte, parece indudable un aumento extraordinario del $\mathrm{CO}_{2}$, gas que se considera produce efecto invernadero por la mayoría de los químicos, aunque no por todos, y según los bioquímicos explica la adicidificación de los océanos y la progresiva desaparición de seres vivos marinos de concha como los foraminíferos (ZEEBE, 2012b). También, como se ha apuntado antes, la disminución de las banquisas polares, glaciares e hielos árticos y de montaña. Y asimismo, habría que considerar, con un mayor peso en los modelos estadísticos proyectivos de los cambios climáticos del que se ha hecho hasta ahora, la influencia fundamental de los ciclos de actividad solar, pues algunos estudios, como los realizados por el MET OFFICE inglés y la Unidad de Investigaciones Climáticas de la Universidad de East Anglia, indican que el incremento de las temperaturas mundiales finalizó ya y que los ciclos solares alcanzarán un mínimo de actividad en el año 2022, que llegará a ser menor incluso que el mínimo de Dalton ocurrido entre 1790 y 1830 o el de Maunder entre 1645-1715 (pequeña edad de hielo). En 2030 se alcanzará el mínimo de Gleissberg según las cadencias y tendencias de los ciclos solares. El Had CRUT3 Dataset es una red histórica con registros mensuales de anomalías en las temperaturas recogidos desde 1850 con un dato de cada cuadrado de $5^{\circ}$ de Lat y Long de la Tierra, tanto en el mar como en tierra firme. Sus datos están siendo trabajados por la MET OFFICE UK y la Universidad de East Anglia del Reino Unido. Disponiendo de potentes SIG's se ha detectado que entre enero y octubre de 2011 hubo un incremento medio de $0,36^{\circ} \mathrm{C}$ sobre la media computada del periodo 1961-1990 y en esto coin- 
ciden bastante con lo deducido por NOAA con datos de satélites. Según estos estudios, desde 1850 cada década ha sido más cálida que la anterior.

Gráfico 3. Coincidencias en los estudios de Met Office (UK), NASA y NOAA del Calentamiento Global

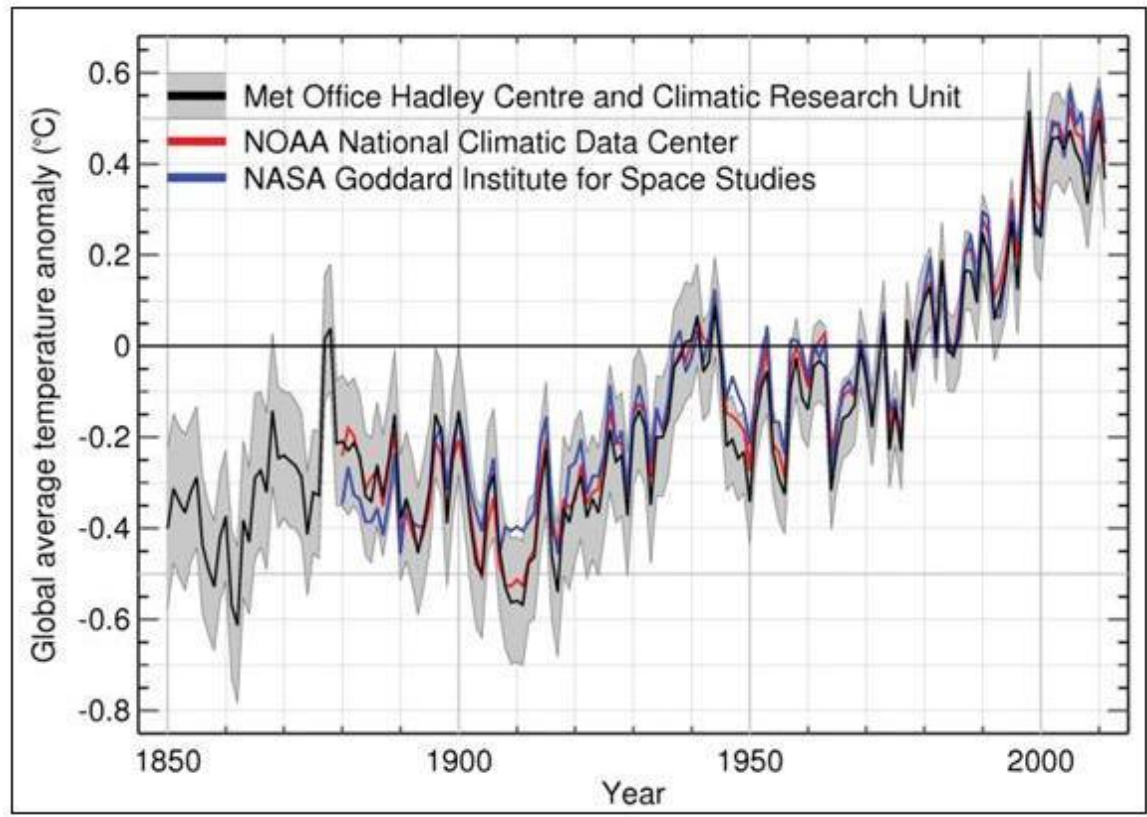

FUENTE: MET OFFICE

Por último, también conviene aclarar el indudable incremento de los impactos económicos y personales producidos por eventos extremos que se achacan al cambio climático, pues también es cierta la extensión de ubicaciones de las poblaciones y de sus actividades con los crecimientos demográficos e inmigratorios en lugares que las convierten en más vulnerables a los riesgos naturales (lechos mayores de ríos, pendientes deslizables, fallas activas, litorales muy bajos etc.).

Todo esto hace que prácticamente no se cuestione ya el calentamiento global y sus máximos en los años 1990, 1996 y 1998 (este último el más elevado de los registrados entre 1979 y 2008) pero sí su atribución causal y, sobre todo, su incidencia concreta sobre cada lugar (GARCíA-SOTO 2002). Por ejemplo, de no haber sido por el análisis de los datos enviados por un grupo de satélites y computados con SIG's por el laboratorio JPL de la Nasa no hubiese sido posible valorar el rápido deshielo en cuatro días que se produjo a mediados del mes de julio de 2012 de una capa muy superficial del 97\% de 
la superficie helada de Groenlandia; fenómeno que inmediatamente fue achacado por el actualismo de la prensa generalista al cambio climático, sin considerar que es un fenómeno que se repite anualmente por la advección de masas de aire procedentes del sur y que hace 150 años se produjo con las mismas características de extensión, como ha sido deducido por estudios paleocriológicos, según ha puesto de manifiesto en algunas entrevistas el físico de la UCLM y miembro del IPCC Manuel de Castro.

Gráfico 4. Curva del calentamiento global

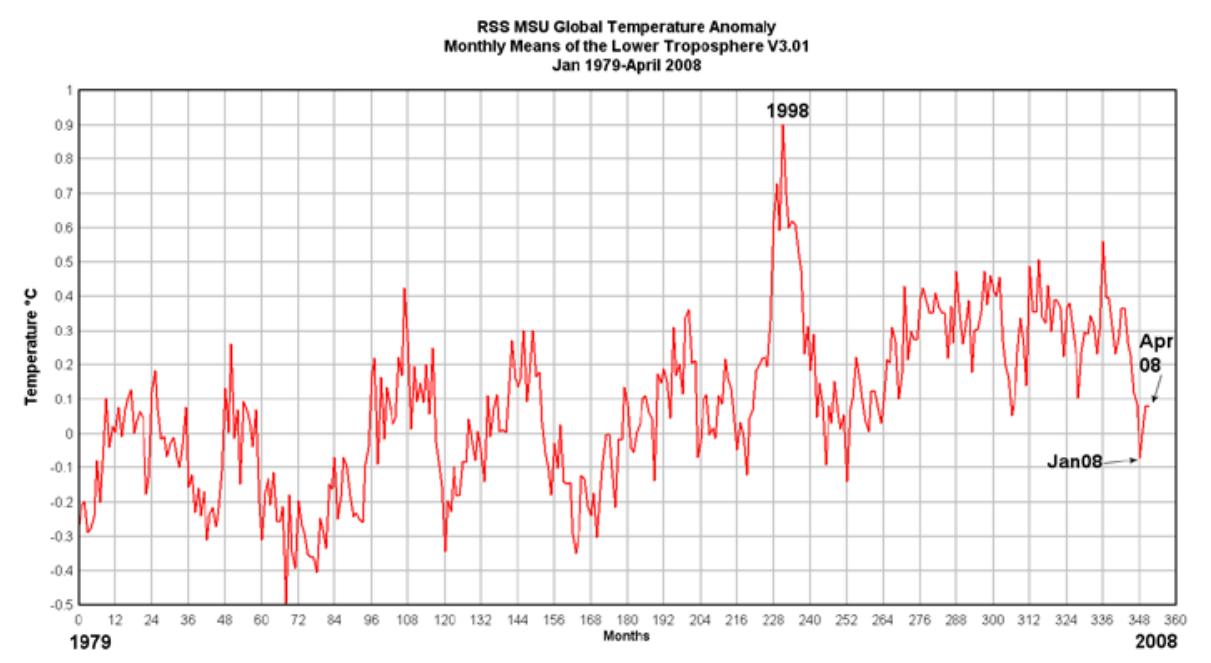

RSS MSU: Remote System Microwave Sound Unit

FUENTE: http://wattsupwiththat.com/2008/05/05/rss-msu-lt-global-temperature-anomaly-forapril-2008-flat/

Así pues, es necesario servirse de otras fuentes de datos y de métodos, además de los clásicos, para conseguir un seguimiento de más detalle, más frecuente y más susceptible de generalizar a todo el planeta los resultados desde puntos concretos. Los principales son las metodologías estadísticas utilizadas y los medios de captación de los parámetros atmosféricos. En ambas, juegan actualmente un papel fundamental las Tecnologías de la Información Geográfica, y de entre ellas los Sistemas de Información Geográfica y la Teledetección. Así se evitará que lo catastrófico radique más que en la realidad en los modelos y simulaciones obtenidos por la escasez de datos, por su interpolación y extrapolación excesivas, y por una mala aplicación de las escalas espaciales y temporales de cada investigación. 


\section{EVOLUCIÓN DE LAS TECNOLOGÍAS DE LA INFORMA- CIÓN GEOGRÁFICA EN RELACIÓN A LA CLIMATOLO- GÍA}

\section{III.1. Cartografía}

A pesar de que desde Estrabón en la Geografía ha existido una preocupación temprana por uno de los factores más determinantes de la variabilidad de la superficie terrestre como son los climas, la falta de datos que discriminaran sus distintos parámetros constituyentes, permitiendo su análisis y su descripción gráfica en mapas, provocó la relegación en su estudio respecto a los demás factores geográficos durante muchos siglos, hasta que se inventaron los aparatos capaces de medir la variación de tales parámetros y su disposición simultánea. Esto último empezó a ser posible con el invento del telégrafo eléctrico en 1837. No es extraño pues que hasta la segunda mitad del siglo XIX, no fuesen creándose las redes meteorológicas que centralizasen en oficinas de servicios estatales los análisis estadísticos y aclarasen los climas locales, regionales y zonales; y de acuerdo a esto, fuese posible su plasmación y descripción cartográfica. Parece que el primer mapa puramente climatológico fue el de vientos dibujado por Hadley a principios del XVIII.

Figura 1 $^{2}$. Mapa de vientos de Hadley

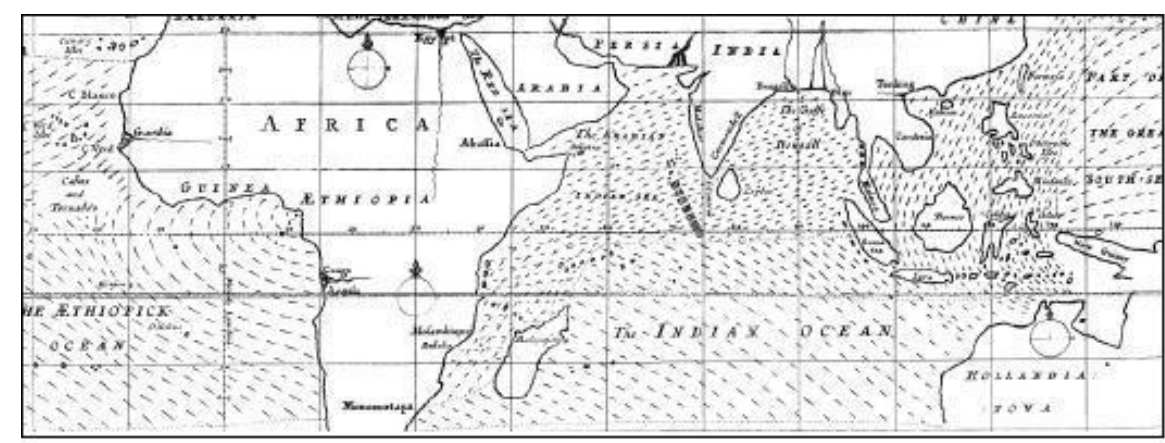

${ }^{2}$ Las figuras 1 a 3 han sido obtenidas en:

www.meteo.fisica.edu.uy/Materias/elementos_met_y_clima/teorico_elementos_met_y clima/EMyChistoriaMET-GVN2012.pdf;

www.tutiempo.net/silvia_laroca/Temas/Historia.htm

www.aemet.es/documentos/es/conocermas/dia_meteorologico/2012/Conferencia_Palo mares.pdf. 
En cuanto a los mapas sinópticos, como es lógico, también fue necesario que existiesen redes de observatorios con datos simultáneos. Así el primero que se publicó fue el que realizó Loomis de una tormenta ocurrida en NE de EE.UU en 1842. En España el primero fue realizado el 1 de marzo de 1893.

Figura 2. Primer mapa sinóptico de Loomis (1842)

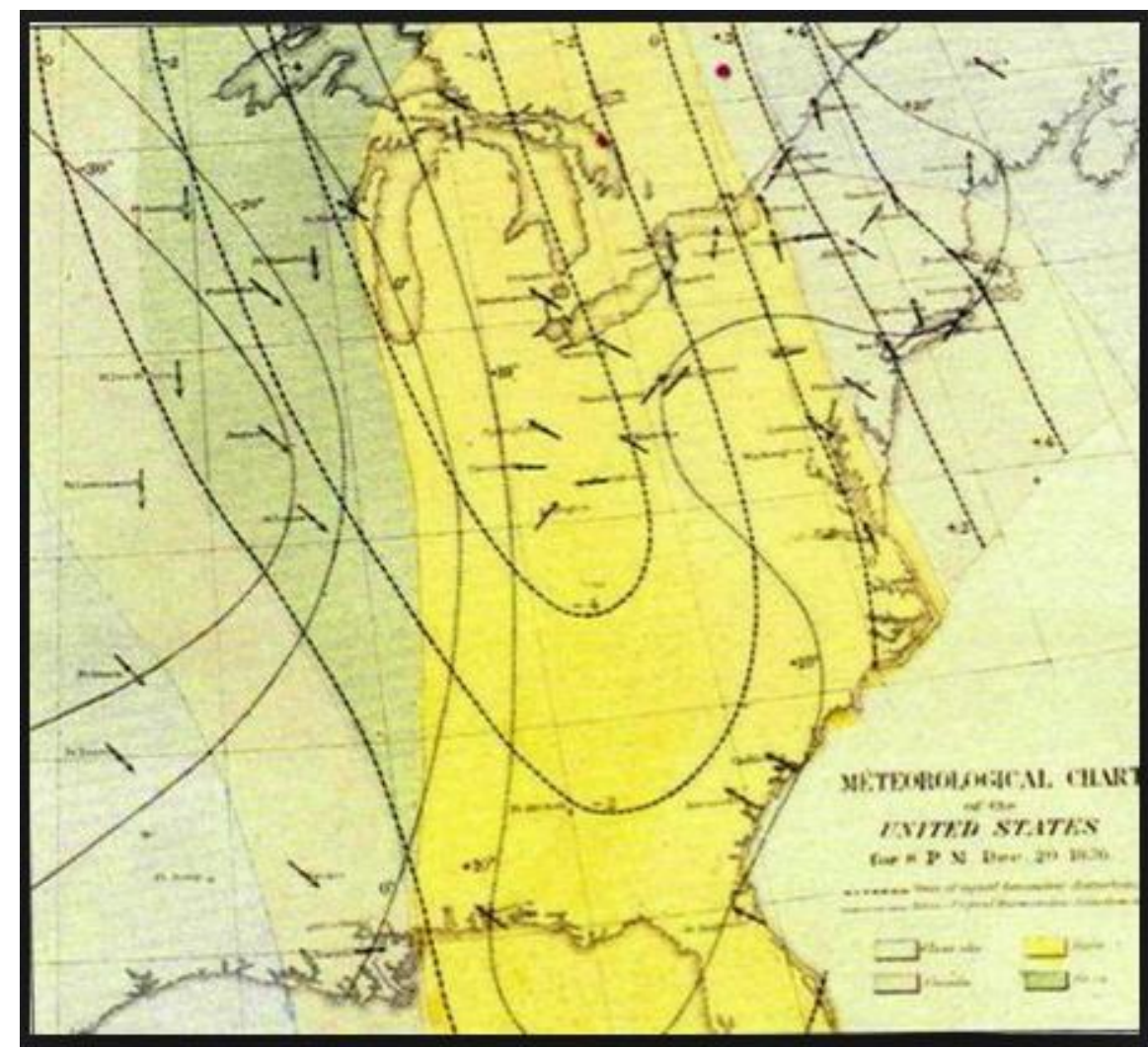

Además de a ser descritos mediante los habituales mapas analíticos y estáticos meteorológicos de carácter sinóptico que describen estados atmosféricos concretos, los climas se prestan a un cartografiado en mapas sintéticos y dinámicos. Esto permite ver de forma gráfica clara como se extienden, hasta dónde y con qué evolución temporal. Generalmente estas descripciones pictóricas se realizan con la diferente coloración de las superficies que comprenden las líneas isopletas de los distintos parámetros estadísticos o sintéticos (temperaturas medias, precipitaciones acumuladas, etc.). La evolución de la cartografía climatológica se acelera y es clara desde que el climatólogo francés del siglo XX Enri-Marcel Gaussen estableció que la gama de color 
azul representaba bien los aspectos de humedad, las de color rojo el calor, los amarillos la sequía, etc; de modo que las combinaciones de estos colores expresasen significativamente características de los climas: violetas (azules más rojos) caracterizan el cartografiado de las zonas ecuatoriales (cálidas y húmedas); anaranjados (amarillos más rojos) los desiertos cálidos; verde (azules más amarillos) a las regiones templadas; etc. Todos los mapamundis se suelen seguir coloreando de este modo. Los mapas climatológicos dinámicos en animaciones móviles permitirán mostrar cambios temporales en los parámetros climatológicos, utilizando anamorfosis exageradas y jugando con nuevos sistemas de proyección cartográfica como, por ejemplo, el de Mark Newman de 2006 (QUIRÓs, 2011).

Figura 3. Primer mapa sinóptico español (1893)

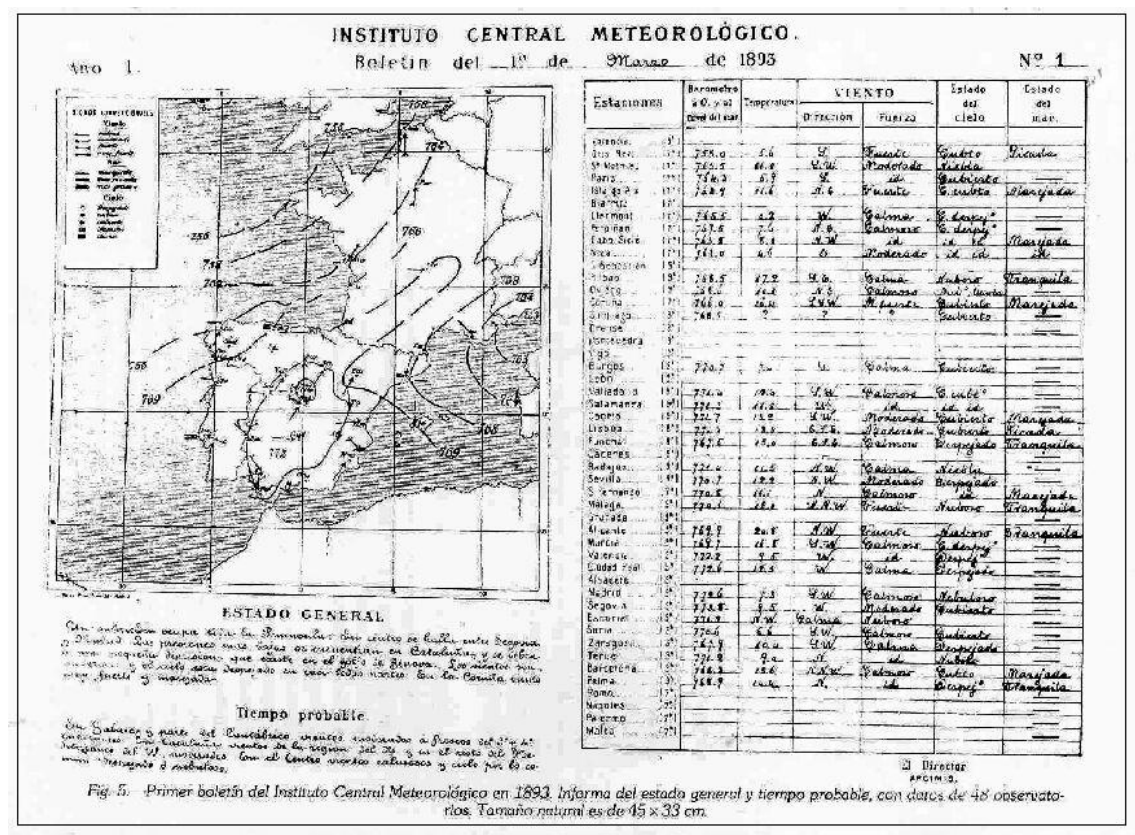

Actualmente, la homogeneización de los datos archivados en distintos servidores de la red Internet, en lo que se denomina red en nube (“cloudnet”), pueden ser accedidos, gestionados y tratados desde cualquier ubicación y ordenador personal con los programas informáticos contenidos en los distintos Sistemas de Información Geográfica, permitiendo colaboraciones e investigaciones conjuntas y simultáneas entre distintos investigadores dispersos. Hoy es posible realizar mapas compartidos incorporando datos homogenei- 
zados desde distintos lugares, como por ejemplo ocurre con la web "eye on earth" de la Agencia de Medio Ambiente Europea; así como utilizar mapas como fuentes para la realización de otros mapas desde distintas páginas "web” (mashups) como, por ejemplo, el proyecto ArcGis On Line que elimina la necesidad de cargar programas SIG en el propio ordenador u otro soporte. El futuro cartográfico general y en particular el climatológico que se avecina es muy potente. La tecnología cartográfica digital, empleada con imaginación pero con rigor, abre nuevas perspectivas a la investigación, proporcionando grandes ayudas a los nuevos métodos para la investigación del cambio climático.

Figura 4. Temperaturas y corrientes oceánicas del mes de enero (Philips'Modern School Atlas-1979)

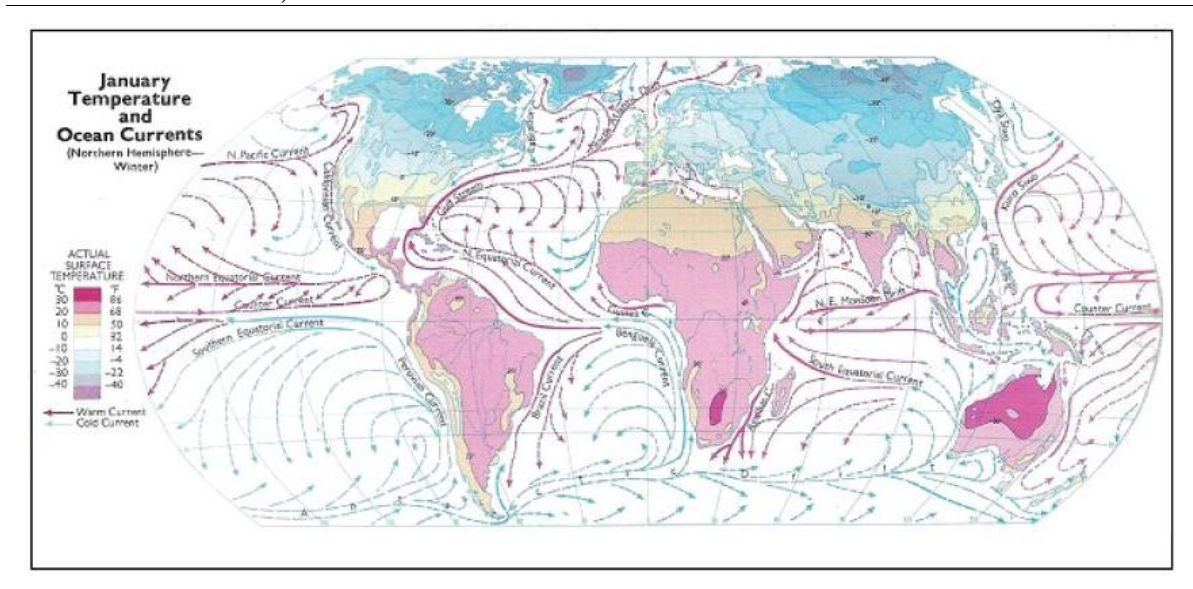

\section{II.2. Teledetección}

Desde que en 1957 fue lanzado al espacio exterior el primer satélite artificial (Sputnik I soviético) el incremento de plataformas espaciales que se han puesto en órbita alrededor de la Tierra ha ido creciendo de año en año de un modo exponencial, de tal manera que se ha llegado a hablar de la ciberelectroesfera. Se han lanzado desde entonces 6.739 satélites artificiales con misiones de distinta naturaleza: telecomunicaciones, televisión, navegación, localización, observación y ciencia. Hoy operan 957 en los que participan 115 países, sin contar los mal denominados microsatélites ni los secretos. En el año 2010 estaban en órbita 3.494 de todos los lanzados desde 1957. Según Matt Amato, ingeniero del Analytic Graphis Inc., actualmente hay unos 13.000 objetos artificiales circunvalando la Tierra que incluyen la basura o chatarra espacial (fragmentos y restos de satélites). De todos estos, 848 proporcionan datos de aplicación a las Ciencias de la Tierra. Uno de los más re- 
cientes es el MKD-FKI-1 lanzado el 22 de julio de 2012 por Rusia, capaz de suministrar datos de salinidad y temperaturas del mar y humedad de la superficie terrestre. El físico suizo Walter Flury, experto en objetos espaciales, ha establecido las siguientes proporciones existentes: $7 \%$ naves operativas; $22 \%$ naves obsoletas; resto de cohetes $17 \%$; objetos para misiones concretas $13 \%$ y fragmentos $41 \%$. La chatarra espacial constituye pues la mayoría de los objetos y ya suponen cierto problema para las plataformas operativas.

Desde el primer momento en que comenzó a estudiarse el cambio climático se asoció y apoyó en la teledetección. La escasa disposición de registros meteorológicos provocaba excesivas extrapolaciones. Esto hizo que fuera necesario ensamblar datos derivados de satélites, observaciones de campo y sondas, mediante algoritmos y modelos. Sirva como ejemplo el proyecto NASA/POWER que utilizó datos de temperatura de Estados Unidos desde 1983 hasta 2004 de 855 observatorios meteorológicos terrestres, computando además sus altitudes y datos complementarios suministrados por satélites artificiales, comparando máximas y mínimas diarias con un valor de correlación $\mathrm{R}_{2}$ de 0,88 y un error cuadrático medio de $4,18^{\circ} \mathrm{C}$; y eso que se evitó la utilización de los situados en regiones montañosas y costeras por su mayor variabilidad (WHITE, 2008).

Cuadro 2. Variables para el proyecto NASA/POWER

\begin{tabular}{|c|c|c|c|}
\hline Variable & Fuente & Periodo de datos & $\begin{array}{l}\text { Periodicidad } \\
\text { de datos }\end{array}$ \\
\hline $\begin{array}{l}\text { Temperatura } \\
\text { máxima, mí- } \\
\text { nima y media } \\
\text { diaria }\end{array}$ & $\begin{array}{l}\text { Gooddard Earth Ob- } \\
\text { serving System } \\
\text { (GEOS) Modelo de } \\
\text { integración V } 4 \text { GEOS } \\
\text { V 5.01 GEOS V } 5.1\end{array}$ & $\begin{array}{l}\text { GEOS V } 4 \text { (Enero } 1983 \\
\text { a Diciembre 2006) } \\
\text { GEOS V } 5.01 \text { (Enero } \\
2007 \text { a Diciembre 2007) } \\
\text { GEOS V } 5.1 \text { (Enero } \\
2008 \text { hasta hoy) }\end{array}$ & $<1$ mes \\
\hline Precipitación & $\begin{array}{l}\text { Satelite y red observa- } \\
\text { torios terrestres. Pro- } \\
\text { yectos TRMM y } \\
\text { GPCP }\end{array}$ & Enero 1997 hasta hoy & $<2$ meses \\
\hline $\begin{array}{l}\text { Radiación so- } \\
\text { lar }\end{array}$ & $\begin{array}{l}\text { Observaciones desde } \\
\text { satélites }\end{array}$ & $\begin{array}{l}\text { Julio } 1983 \text { a Junio 2006; } \\
\text { Julio } 2006 \text { hasta hoy }\end{array}$ & $<1$ mes \\
\hline $\begin{array}{l}\text { Temperatura } \\
\text { del punto de } \\
\text { rocío }\end{array}$ & $\begin{array}{l}\text { GEOS V } 4 \text { GEOS V } \\
5.01 \text { GEOS V } 5.1\end{array}$ & $\begin{array}{l}\text { GEOS V } 4 \text { (Enero } 1983 \\
\text { a Diciembre 2006) } \\
\text { GEOS V } 5.01 \text { (Enero } \\
2007 \text { a Diciembre 2007) } \\
\text { GEOS V } 5.1 \text { (Enero } \\
2008 \text { hasta hoy) }\end{array}$ & $<1$ mes \\
\hline
\end{tabular}


Hasta mediados de los años noventa, para seguir el cambio climático desde el espacio exterior había que hacerlo con datos suministrados por satélites diseñados para otros fines; hasta que finalmente dentro del NPOESS (Programa del Sistema de Operaciones de Satélites Ambientales) desde 1998 se dispone de datos suministrados por sensores como MODIS (ModerateResolution Imaging Spectroradiometer), ASTER (Space Termal Emisión and Reflectance Radiometer), MISR (Multiangle Imaging SpectroRadiometer), CERES (Cloud and Earth's Radiant Energy System) y otros, diseñados especialmente para el seguimiento del cambio climático y sus efectos sobre la superficie terrestre, que están portados por plataformas como el satélite EOS AM1 que funcionan en coordinación con otras anteriores más dedicadas al estudio de los usos de suelo como LANDSAT-7. Se trataba de proporcionar un sistema de muestreo multiescala del planeta con captaciones de sectores de 250, 500 y 1000 metros de lado; a la vez que se diseñaban algoritmos matemáticos para tratar los datos de radiación y convertirlos en parámetros físicos, meteorológicos y climatológicos. El grupo de científicos de Ciencias de la Tierra (MODLAND) dentro del Programa EOSPSO (Oficina del Proyecto Científico del Sistema de Observación de la Tierra www.eospso.gsf.nasa.gov-) establecieron distintos niveles de productos de superficie (LPL's) que proporcionaban técnicas para conseguir datos de los siguientes parámetros fundamentales: reflectancia espectral de la superficie terrestre; albedo y BRDF (función de distribución de reflectancia bidireccional); temperatura de suelo o brillo en mar y en superficie terrestre; índices de vegetación; índices de área foliar (LAI) y FPAR (fracción de radiación absorbida para la fotosíntesis activa); fuegos activos y áreas quemadas; cubiertas de nieve, lagos helados, glaciares y banquisas; usos de suelo y sus cambios; y validaciones, verificaciones y precisiones de los datos. La OMM añadió a éstos clorofila en el océano; aerosoles; ozono y otros gases traza; y cubiertas nubosas y sus tipos.

Uno de los problemas o dificultades en teledetección que siempre han preocupado, han sido tenidos en cuenta y han tenido una evolución clara, especialmente en sus aplicaciones climatológicas, ha sido el de la conversión de radiaciones en parámetros físicos y sobre todo el de las resoluciones: qué, cómo y cuanta información suministra la unidad mínima información de la superficie terrestre (píxel) que recoge un sensor; también la degradación de la información de tipo gaussiano tridimensional que se produce hacia los bordes del píxel. Se distinguen cinco tipos de resoluciones que han evolucionado tecnológicamente mucho pero siempre hay que tener en cuenta: $A$ ) Resolución espacial o extensión de la superficie terrestre que cubre un píxel y se suele establecer mediante una función matemática (MTF -modulation transfer function-); la Nasa recomienda para su medida utilizar el EIFOV 
(Effective Instantaneous Geometric Field of View), cuya dimensión de correspondencia con la medida en la superficie terrestre responde a la frecuencia (1/ $\mu$-inverso de la longitud de onda a la que es sensible el sensor-), para la cual el valor de la MTF es 0,5. B). Resolución espectral que viene determinada por la SRF (Spectral Responsebility Function) y define la sensibilidad con la que el sensor detecta radiancia para cada longitud de onda, y tiene valor nulo por encima y debajo de los umbrales para los que ha sido diseñado. C) Resolución radiométrica que indica la sensibilidad del sensor a la intensidad de la radiación o mínimo cambio de intensidad que puede detectar; que antes se medía con el número de bits con el que el sensor codificaba esta información y actualmente es preferible utilizar la relación NEDT/ NEDR (Noise Equivalent Diferencial Temperatura/ Noise Equivalent Diferencial Reflectance). D) Resolución temporal o ciclo de repetición que se mide mediante el tiempo que tarda el sensor en volver a cubrir una localización determinada, manteniendo la misma geometría de captación que establece el ancho de barrido (Swath). Esta resolución es muy difícil de establecer cuando los sensores son de ángulo de captación regulable o de capturas solicitadas que obliga a diferenciar el concepto de "tiempo de revisita" desde distintos ángulos. Actualmente está muy relacionada con E) Resolución angular o número de ángulos desde los que se captan las radiaciones procedentes de un espacio terrestre equivalente a un píxel. Una integración de todos estos cinco tipos de resolución viene expresada en el índice de calidad RQI (Relative Quality Index) que permite la comparación entre distintos tipos de sensor (CALLE, 2011).

Los datos que suministran las plataformas espaciales que actualmente pueden venir asociadas a la información de su localización y es obtenida de manera auxiliar con las constelaciones de satélites GPS, Glonass, Galileo, etc. son fuente de información para detectar y analizar cualquier riesgo natural, incluidas las alteraciones climáticas. Existen series temporales, que en algún caso cubre los últimos 50 años, de información almacenada en los organismos y empresas que manejan y explotan los datos obtenidos en el pasado y en la actualidad por los distintos satélites.

Muy pronto (1959) fueron diseñados los primeros exploradores multiespectrales que permitieron captar fenómenos que emiten radiaciones muy por encima y por debajo del espectro radiométrico visible para el ojo humano y, por tanto, facilitaban hacer el seguimiento de meteoros como las concentraciones de vapor de agua o las temperaturas. En 1966 Estados Unidos puso en órbita el primero de los que se conocen como geoestacionarios que pertenecía a un programa de seguimiento repetido con una alta frecuencia en la toma de datos, denominado en un principio ATS y luego pasó a denominarse 
SMS, para en 1975 adquirir el nombre con el que hasta ahora es conocido: GOES (Geostationary Operacional Enviromental Satellite). Es el equivalente de otros proyectos de otros países como el europeo METEOSAT que nació en 1972 controlado por EUMESAT (European Organitation for Explotation of Meteorogical Satellites) y hoy pertenece a la Agencia Espacial Europea (ESA). Este último, mediante su ciclo de giro coordinado con el del planeta suministra información del hemisferio captable desde una posición cero grados de latitud y cero de longitud desde la vertical del Golfo de Guinea a $36.000 \mathrm{~km}$ de la Tierra. Estos dos grandes programas meteorológicos satelitales completan información de todo el planeta junto al programa meteorológico japonés MTSAT GMS (Geostationary Meteorogical Satellite); el ruso GOMS (Geostationary Operational Meteorological Satellite); el hindú Kalpana de INSAT (India Nacional Satellite); el chino FengYun (FY-2); y otros hasta completar una constelación de 16, coordinados en sus datos por la OMM con su pionero y temprano programa GARP (Global Atmospheric Research Programme). Actualmente el programa MSG europeo (METEOSAT8 y 9 de Segunda Generación), que desde marzo 2006 ya maneja el noveno satélite geoestacionario desde su creación, suministra información cada 15 minutos de un sensor SEVIRI (Spinning Enhaced Visible and Infrared Imagen) dividido en 12 intervalos del espectro electromagnético que radia la atmósfera y la superficie terrestre: tres en el visible, dos en los de vapor de agua, cuatro en el infrarrojo próximo y medio, y tres en el infrarrojo térmico, para poder seguir todos los fenómenos meteorológicos que se producen de polo a polo en el Atlántico, Europa y África ${ }^{3}$. Una característica de este programa es que a medida que se lanza un nuevo satélite de la serie se desplaza el anterior en la misma órbita hacia el este; lo que permite tener información de Asia y el Pacífico, además de las que suministran las administraciones de los países correspondientes a esas longitudes geográficas.

En 1960 en la NASA (USA) nace el primer proyecto de un satélite con órbita cerrada denominado TIROS-1 que se diseñó para seguimiento meteorológico. Fue el pionero de lo denominados heliosíncronos de órbita polar. En 1978 este proyecto pasó a denominarse con el nombre de la administración estadounidense que lo explota (NOAA -National Oceanic and Atmospheric Administration-) con parejas de satélites en posiciones complementarias que giran en direcciones opuestas, una ascendente y otra descendente respecto al plano ecuatorial; de manera que pasan por la vertical de cada punto terrestre

\footnotetext{
${ }^{3}$ El Meteosat-6 nunca entró en funcionamiento. Hasta el Meteosat-7 la frecuencia de datos suministrados era cada 30 minutos y cubría 4 canales del espectro: dos en el visible, uno en el infrarrojo térmico y otro en el infrarrojo medio denominado de vapor de agua (WV) para captar la alta troposfera.
} 
a primera hora de la mañana y primera de la tarde (momentos que suelen coincidir con aquellos en los que se producen las temperaturas mínimas y máximas diarias en cada lugar). En julio de 2006 se puso en órbita el primer satélite meteorológico europeo de órbita polar: METOP-A, lanzado por la Agencia Espacial Europea bajo el programa EPS (Eumesat Polar System) que porta un sensor radar, otros meteorológicos y de apoyo a la navegación y a los rescates. Participa junto EE.UU. en la IJPS (Inicial Joint Polar-orbity Satellite System) y se coordina con el NOAA-18; aunque en breve lo hará con el METOP-B que ha sido lanzado en septiembre de 2012 en la misma órbita que el A y con los mismos fines: estudios de temperaturas, humedad, velocidad e intensidad de vientos. En 2016 será lanzado el METOP-C y para 2020 se prevé que habrá otros seis satélites de segunda generación. Otros proyectos que poseen satélites con este tipo de órbitas bajas y portan sensores de todo tipo, algunos especialmente diseñados para seguir el cambio climático, son el EOS (Hearth Observing System) de USA, Japón y Canadá que hoy controlan los programas Landsat-7, Terra, Aqua, Jasón-1, Ice-sat, Cloud-sat; ERS (European Remote Sensing); ENVISAT (Enviromental Satellite); SPOT (Satellite Pour l'Observation de la Terre); CBRS (China BRaSil); SAUCOM (Agencia Argentina para la Observación de la Tierra); OceanSat; CartoSat; ResourceSat; NPOESS (National Polar Orbiting Enviromental Satellite System) que controla los satélites NOAA; DEIMOS-1 español; etc.. Los principales sensores entre otros que portan estos satélites de aplicación climatológica son: AVHRR (Advanced Very High Resolution Radiometer); ASCAT (Advanced Scatterometer) para el estudio de vientos en todas las capas atmosféricas; ATOVS de infrarrojos y microondas; IASI (Infrared Atmospheric Sounder Interferometer) para seguimientos de humedad y temperatura; GRASS (GNSS Receiver for Atmospheric Sounder) o GOME (Global Ozone Monitoring Experiment).

La Agencia Espacial Europea (ESA) está participada por 18 países: Austria; Alemania; Bélgica; Chequia; Dinamarca; España; Finlandia; Francia; Grecia; Holanda; Irlanda; Italia; Luxemburgo; Noruega; Reino Unido; Suecia; Suiza y en algunos proyectos Canadá. Tiene cinco centros en los que trabajan unas 2.000 personas. Las misiones o proyectos que actualmente controla con diferentes aplicaciones y objetivos son: SMOS; SWARM; GOCE; CryoSat-2; ADM-Aeolus; Hearth Care; METEOSAT; Sentinel 1 a 5; ERS 1 y 2; ENVISAT; ESAfire-cci y EO. En los próximos diez años se lanzarán al espacio veinte satélites con sensores especialmente diseñados para el seguimiento del cambio climático: ECV (Essential Climate Variables) para colaborar en el UNFCCC y el IPCC. (OLIVIER 2011). Dentro del proyecto ESAfire_cci de seguimiento de incendios forestales, también servirá al estudio climatológico ECVGCOS con los sensores ATRS, VGT y MERIS que 
permitirán generar series largas y consistentes de variables climáticas esenciales para las modelizaciones del clima y de los ciclos de carbono (Chuvieco 2011).

A la vez existen redes de satélites geoestacionarios de telecomunicación comerciales para la distribución de los datos satelitales, aunque cada vez son más sustituidas por Internet, como la EumetCast con estándar DVB (Digital Video Broadcast) y centro de distribución en Darmasdt (Alemania).

Las agencias espaciales nacionales e internacionales colaboran para el estudio conjunto del cambio climático con sus satélites de última generación que soportan radares, sondas, espectrómetros, scatterometers, etc. mediante los que se puede calcular un amplio rango de variables fisicoquímicas y biofísicas que intervienen en los procesos climatológicos; especialmente los denominados de órbita baja (LEO -Low Hearth Orbit) entre los 200 y los 1200 $\mathrm{km}$, y sobre todo los heliosíncronos de órbita polar que giran a una altura sobre la Tierra de $800 \mathrm{~km}$. Entre los más destacados se encuentran desde noviembre de 2009 el SMOS (Soil Moisture and Ocean Salinity) o el CryoSAT (abril de 2010) de la ESA; el H2A japonés desde enero de 2009 o el SAC-D argentino en orbita desde junio de 2011; el NPP norteamericano desde octubre de 2011, etc... Sólo en 2008 fueron puestos en órbita terrestre 18 satélites para vigilar el clima y desde abril del año 2012 y hasta 2014 el proyecto Pegaso para el estudio de aerosoles y el clima sobre Europa dispone de un satélite propio (LAS HERAS, 2010). El proyecto europeo SMOS, que además del satélite controla boyas oceánicas a la deriva, intenta compensar la escasa disponibilidad de datos de humedad en suelo y de la salinidad en los océanos que son fundamentales para estudiar la variabilidad climática y los ciclos del agua. Se basa fundamentalmente en la radiación que depende de las propiedades dieléctricas del suelo y del mar, es detectada por sensores y transformada como parámetro del contenido de agua en los suelos. La salinidad influye en las distintas densidades del agua y, por tanto, sobre las cinturones de circulación oceánica. Este satélite posee un sensor interferométrico multiangular que proporciona una precisión del $4 \%$ en el cálculo de la humedad, tiene una resolución espacial de $50 \mathrm{Km}$ y un periodo de revisita en cada lugar de tres días. Para la determinación de la salinidad la precisión es de 0,1 unidades y la resolución es de 100-200 Km. Tiene un sensor radar con antena de apertura sintética (MIRAS Microwave Interferometric Radiometer Using Aperture Synthetic) con una resolución angular entre $0^{\circ}$ y $60^{\circ}$, resolución espacial de 20-70 Km y revisita cada 1-3 días; este tipo de sensor hasta ahora solo se aerotransportaba en aviones. En España los centros dependientes o que colaboran con este proyecto son el ESAC (European Space Astronomy Center de Villafranca del Castillo (Madrid); el CP34 (Centro de proceso de 
datos SMOS de niveles 3 y 4 -productos L1c-; y el SMOSBEC (Barcelona Expert Centre on Radiometric Calibration and Ocean Salinity) (FONT, 2011).

España se incorporó muy pronto a la explotación de datos satelitales de administraciones extranjeras aunque mucho más tarde lo hiciera al lanzamiento y operación de satélites propios; así es que en ese sentido puede considerarse a nuestro país pionero en este campo. En 1986 se realiza la primera reunión y la constitución de la Asociación Española de Teledetección (AET) que hasta hoy ha publicado 37 números de su revista (a la que se puede acceder directamente en su web: aet.org.es), donde se recogen muchos artículos de investigaciones sobre climas realizadas con datos satelitales, destacándose los de cálculos de la temperatura de suelo y aire (CASELLES, 1997; NiCLÓS, 2011; REDONDO, 2011; etc..). Sus Congresos son bianuales. En septiembre de 2011 en Mieres del Camino (Asturias) se reunió su XIV Congreso bajo el lema "Teledetección, Bosques y Cambio Climático" y el próximo (XV) se celebrará en Torrejón de Ardoz (Madrid) organizado por INTA en octubre de 2013, bajo el lema "Sistemas Operacionales de Observación de la Tierra”.

La importancia que oficialmente se da en España a la teledetección en general no solo viene expresada en la temprana explotación y utilización de sus datos, sino en la existencia de un Plan Nacional de Teledetección (PNT) que se ha marcado como objetivos facilitar la utilización de imágenes y datos derivados, disminuir los costes económicos y aminorar los plazos entre las captaciones y sus usos. Su coordinación general la realiza el Instituto Geográfico Nacional del Ministerio de Fomento y participa INTA del Consejo Superior Geográfico del Ministerio de Defensa. Sus antecedentes fueron el CORINE land Cover de 1990 y otras iniciativas como el PNOA (Plan Nacional de Ortofotografía Aérea) de 2004, el Image \& CLC 2006 y 2009, el GMS, Geoland, etc... La primera asamblea se celebró en octubre de 2007 en Maspalomas (Gran Canaria) y en 2008 se formó una comisión especializada de observación del territorio. En 2010 su presupuesto económico fue de 2.626.469 euros. Fue revisado el Plan (PNT versión 3.0) en junio de 2011. Participan 42 organismos de las Comunidades Autónomas, 47 de la Asociación de Geógrafos Españoles (AGE) y 66 Universidades. Utiliza informaciones procedentes de SPOT-5(HRG) y Rapid Eye para datos de alta resolución espacial; Landsat-7 y el español DEIMOS-1 para media resolución; y MODIS, MERIS y VEGETATION para la baja resolución espacial. Para series históricas almacena datos de España procedentes de los Landsat 1 a 7 (MMS, TM y ETM+) y los NOAA. En el futuro está previsto utilizar datos de los satélites INGENIO y SPOT 6-7 para la alta resolución; Sentinel-2 y Landsat Data (continuity misión) para la media; y Sentinel-3 para la baja. Se 
tiene la intención de ir agrupando la información dispersa actualmente en las distintas infraestructuras de datos espaciales (INSPIRE, IDEE e IDEs'Autonómicas) y los distintos servidores de imágenes (WMS, PNOA, Iberpix, SigPac). En 2010 se finalizó el mosaico de alta resolución y plena corrección geométrica (2,5 metros) que cubría toda España con SPOT-5. Actualmente se recogen 250 imágenes al año del SPOT y 800 del Landsat5 en formato ftp de internet, Deimos (sensor SLIM6 de 8/10 bits, cada 3-4 días, 22 metros de resolución espacial) Modis y Meris (VILLA, 2011).

En el aspecto puramente tecnológico, además del proyecto y satélite DEIMOS con centro de operaciones en el Parque Tecnológico de Boecillo (Valladolid) y en el futuro también en Puertollano (Ciudad Real), existe un Programa Nacional de Observación de la Tierra desarrollado por el Centro para el Desarrollo Tecnológico Industrial del antiguo Ministerio de Ciencia e Innovación, cuyo objetivo es el desarrollo coordinado de la financiación y explotación del satélite óptico INGENIO (SEOSAT) con una resolución de 2,5 metros en el pancromático y de 10 metros, con un ancho de escena de 60 Km y una cobertura de toda España de 4 veces al año; y también del de microondas con radar de apertura sintética PAZ (SEOSAR/PAZ) que suministrará 200 imágenes radar diarias en polarización vertical y horizontal. Será el primer proyecto europeo que cubra ambos espectros rariométricos. El operador español será HISDESAT/INTA y tendrá un uso simultáneo civil y militar. Se espera que el PAZ esté operativo en 2013 y el INGENIO en 2014 (LOMBA, 2011).

Como se puede comprobar, se trata de una auténtica carrera espacial con cada vez más asignaciones presupuestarias que se espera vayan aumentando, pues cada euro invertido en actividad espacial reporta a la economía de un país entre 4 y 20 veces su valor (DUQUE, 2012). Esto va a permitir disponer de una cantidad ingente de datos cuyas Bases sólo serán posible manejar con los Sistemas de Información Geográfica.

\section{II.3. Sistemas de Información Geográfica}

Ya en los primeros noventa del siglo pasado se comprendió que debía ser la Geografía como ciencia integradora una de las principales que estudiase el cambio climático y sus impactos, por su estructura global y su carácter medioambiental. También se entendía que la gran cantidad de datos y sus distintas escalas espaciales y temporales hacían completamente imprescindibles herramientas SIG (Sistemas de Información Geográfica) que permitiesen integrar las fuentes, homogeneizarlas y compactarlas, asociar cada dato climatológico a su situación y localización y a otros factores geográficos influyentes sobre él; así como que dispusiesen de métodos estadísticos avanzados in- 
formatizados, sistemas gráficos muy evolucionados e inteligencia artificial. También se hacían necesarios sensores electro-ópticos en los satélites que permitiesen análisis globales. Se buscaba eliminar la inabarcabilidad del tratamiento de los datos, especialmente en su tratamiento y archivado, encontrando un equilibrio entre las necesidades y los manejos, y entre el detalle, la precisión y la inversión económica informática. Estas necesidades fueron motivando iniciativas y progresos mundiales auspiciados especialmente por la ONU como el GRID (Global Resources Information Database). En 1985 ya se hablaba de la necesidad de una red de bases de datos medioambientales y se diseñó el programa CORINE (Coordination, Information and Enviroment) y el año siguiente el IGBP (Internacional Geospher Biosphere Program) (CHUVIECO, 1992).

La compleja interdisciplinariedad del estudio del cambio climático, sobre todo desde que el AR4 lo atribuye inequívocamente a la actividad humana en base a que entre 1985 y 2000 se han producido once de los doce años más cálidos del siglo veinte, hace que el lábil dinamismo del fenómeno sea objeto claro de estudio de la Geografía por su dedicación a la situación de la superficie terrestre y a los datos en el presente; también de la Geología para deducir tendencias desde la paleoclimatología; y de la Economía y la Historia por sus afecciones y causas; además de otras disciplinas científicas básicas, como la Física, la Química o la Biología (MARTIN-VIDE, 2008).

Ante la percepción generalizada del cambio climático, la Organización Meteorológica Mundial (WMO) y el Programa Ambiental de Naciones Unidas (UNEP) crearon en 1988 el IPCC (Intergubernamental Panel on Climate Change) con tres grupos de trabajo: el IPCC I (WG I) evaluaría los aspectos científicos físicos de los parámetros climatológicos; el IPCC II (WG II) que evaluaría las vulnerabilidades socioeconómicas y naturales en las posibles hipótesis y supuestos del cambio climático, buscando interrelaciones entre tales vulnerabilidades y las necesarias adaptaciones que condujesen a desarrollos sostenibles de los distintos sectores económicos y regiones; y el IPCC III (WG III) que propondría las medidas para mitigarlo, evaluando costos y beneficios, y buscando soluciones con los instrumentos y medidas políticas apropiadas. Además de su dedicación común al cambio climático los tres grupos se encargarían de perfeccionar y desarrollar metodologías internacionales y sus softwares asociados para los cálculos. El TGICA (Task Group on Data and Scenario Support for Impacts and Climate Análisis) fue creado para la coordinación entre los modelos climáticos y las evaluaciones de sus impactos.

La mesoescala y la escala local son fundamentales para valorar con precisión los matices y diferencias en la afectación de los impactos del cambio 
climático, y son grandes los esfuerzos económicos y técnicos para la dotación y creación de redes de observatorios en dichos ámbitos, aunque aún son insuficientes. La OMM ha puesto en marcha un programa mundial de investigación meteorológica (PMIM) con el fin de pasar la escala temporal de predicción meteorológica precisa a periodos mensuales, pero todavía en muchos lugares las redes de observatorios no son lo suficientemente densas. Dentro de este programa existe el experimento THORPEX (experimento de investigación y predictibilidad de los sistemas de observación). Japón tiene una red a media escala denominada JMA. Y la más avanzada es la red MESONET norteamericana que está automatizada y con la que se pretende conseguir densidades de una estación cada 2 a $40 \mathrm{Km}$ que dé información cada 1 a 15 minutos. Lo ideal es que trabajen en tiempo real, mediante instrumentos autónomos (alimentación con paneles solares) de captación de meteoros con memoria propia y transmisión de datos en tiempo regular. En realidad es muy costosa económicamente por lo que son redes a constituir a largo plazo, como ASOS o AWOS que actualmente poseen estaciones separadas entre sí entre 50 y $100 \mathrm{Km}$ y envío de datos cada hora. Redes de este tipo existen en los estados estadounidenses de Florida, Ohio, Utah, Oklahoma, Texas, Missouri, y Carolina del Norte. En todo caso, las cantidades de datos que hay que tratar parece un trabajo imposible sin contar con algún programa sostenido sobre un sistema de información geográfica.

En España, la aportación de la Agencia Española de Meteorología (AEMET) a la acción COST ESO 601 es el Banco Nacional de Datos Climatológicos (www.aemet.es/idi/clima/registrosclimaticos) que cubre desde los datos de precipitación de enero de 1805 del Observatorio Astronómico de San Fernando (Cádiz), siguiendo por los de los 50 observatorios meteorológicos que funcionaban en España en el siglo XIX, hasta llegar hoy a disponer de una base de datos Oracle en la que se recogen los datos de 700 estaciones meteorológicas automáticas. Durante todo este tiempo los cambios en la ubicación de las estaciones, junto a las renovaciones de los instrumentos de medida y sus calibraciones por la evolución tecnológica, y las transformaciones en los usos de suelo de los entornos de los observatorios han provocado faltas de homogeneidad de los datos en las series que a veces se solapan con la propia variabilidad real de la señal climatológica. Esto ha motivado la creación y el desarrollo por la propia agencia en lenguaje estadístico "R" del software denominado "CLIMATOL". Este software permite el tratamiento automático de las series climatológicas, la corrección de las faltas de homogeneidad, y el rellenado de saltos y ausencia de datos. Proporciona valores medios, quintiles, tendencias y otros parámetros estadísticos para el periodo de tiempo que se desee. Ha permitido estudiar las series españolas de precipitación, temperatura, radiación solar e irradiación relativa, elabo- 
rando valores de referencia para el periodo crítico 1981-2010. También se han tratado con este software las series mensuales del proyecto ECAD (European Climate Assesment and Dataset) de la red de servicios europeos EUMETNET.

Las puras técnicas estadísticas utilizadas hasta ahora en Climatología han llegado a ser insuficientes para el estudio preciso y el seguimiento del cambio climático, pues para la generalización espacial de las muestras puntuales de los parámetros climatológicos sobre el espacio geográfico es fundamental el recurso al concepto de "variable regionalizada" que aporta la geoestadística y es una función matemática que adopta cierto valor en cada una de las coordenadas espaciales $\mathrm{x}, \mathrm{y}$, z. Porque los datos en general no sólo presentan una distribución en el espacio geográfico, sino una relación entre ellos en función de su proximidad o lejanía, es decir una correlación espacial y una dependencia de otros factores geográficos perfectamente cuantificables actualmente (formas de relieve y altitud, pendientes, vegetación, usos de suelo, hidrología, etc..). Por esto, se tienen que conjugar dos propiedades generales de los datos, aparentemente contradictorias entre sí. Por una lado, su carácter general estructurado y, por otro, su apariencia errática, aleatoria y local que presenta una variación casi impredecible de un lugar a otro y lleva a una imprecisión en los resultados de las investigaciones, produciendo errores considerables en las estimaciones. El presupuesto básico de la geoestadística consiste en considerar que el valor de un parámetro en un lugar es resultado de un proceso aleatorio con una distribución regular y concreta. Su objetivo es deducir un modelo operativo teórico (variograma teórico) desde una información muestral espacialmente discontinua para delinear la imagen continua interpolada y extrapolada más probable de un fenómeno. A causa de estas características los automatismos informáticos en los estudios climatológicos no son recomendables, y debe ser el investigador y sus conocimientos geográficos el que controle todo el proceso geoestadístico con el apoyo en un SIG. Muchos de los sistemas de información geográfica generalistas de los actualmente existentes contienen módulos informáticos que lo permiten, y han propiciado muchos estudios climatológicos a escalas medias y locales en los que se deducen tendencias espaciales de los parámetros dependientes, considerando variables independientes que influyen sobre sus valores (Quirós 2001). Los desarrollos temporales de cualquier fenómeno climatológico también pueden seguirse y extrapolarse con técnicas desarrolladas por otros módulos contenidos en los SIG's, como por ejemplo las técnicas estadísticas de Markov. También se pueden atribuir las probabilidades de existencia de un meteoro en un lugar, así como la ocurrencia de un fenómeno meteorológico y su integración temporal que permitan definir un clima y sus cambios, mediante los módulos informáticos que se basan en la lógica 
borrosa (fuzzy logic). Otra gran aplicación es la modelización de la diferencial afectación del cambio climático global sobre los climas locales y microclimas, mediante la utilización con un SIG de los archivos digitales de las topografías con los modelos digitales del terreno y de las elevaciones (MDT y MDE); con ellos se obtienen índices de exposición solar que esclarecen las insolaciones potenciales sobre los lugares. Y desde luego, los SIG's ayudan a cartografiar de forma muy eficiente todos los resultados de los análisis climatológicos. Los límites de las aplicaciones climatológicas con los SIG’s son tan amplios e indefinidos que más bien se encuentran en las capacidades y conocimientos que aporten los climatólogos.

\section{CONCLUSIÓN}

La realidad climatológica se encuentra en cambio constante. Con la profusión creciente de los mismos, muchos sensores y satélites no sirven o no están diseñados ex profeso para estudiar los cambios en los climas y están mal coordinados. Muchas veces los algoritmos para los cálculos de los parámetros no son lo suficientemente robustos, precisos, flexibles y fiables y hay que someterlos a controles no demasiado automáticos para que sus derivaciones permitan evitar errores excesivos. Muchos de los procesos y métodos contenidos en los sistemas de información geográfica requieren alta especialización para aplicarlos correctamente. Generalmente las licencias de uso prohíben expresamente no solo la cesión de los datos originales sino incluso de las informaciones derivadas de los mismos ("added value products"). Muchos formatos de datos son obsoletos y poco útiles. Los datos de los observatorios meteorológicos son cada vez más caros, más difíciles de obtener brutos y en tiempo real. Habitualmente los registros del pasado también son de escasa calidad y difíciles de obtener. Todas estas trabas deben ser superadas, pero se dispone ya de los medios tecnológicos avanzados para hacerlo.

El conocimiento del cambio climático no lo pueden dar visiones individuales, propias y personales, sino las colectivas apoyadas en muchas observaciones empíricas sobre los datos objetivos suministrados por las estaciones meteorológicas y sobre todo por la teledetección, y tratados con recurso a los sistemas de información geográfica para plasmarlo en los mapas. La revolución tecnológica en estos últimos diez años permite la mejora técnica en la obtención y el manejo de los datos climatológicos y va a incrementarse en los próximos años a un ritmo cada vez más intenso, con la ventaja de que no se producirá un incremento de costes económicos del mismo nivel, a causa de las características generales de estas tecnologías: a) la intensa y barata permeabilidad geográfica; b) el que el desarrollo económico de cada país ha dejado de ser un obstáculo insalvable para su disposición y uso; y c) la cada vez más precisa normalización y estandarización del contenido y el formato 
de los datos climatológicos favorecerá la integración y la interconexión de bases de datos muy grandes y dispersas. Estas tres características permitirán la internacionalización de la investigación con lo que se denomina "computación colaborativa” y la consiguiente desaparición o al menos la aminoración de controversias, facilitando acuerdos internacionales que mitiguen los impactos medioambientales, económicos y sociales del cambio climático.

\section{BIBLIOGRAFÍA}

ALEXANDER, L.V. et al. (2006): «Global observed change in daily climates extremes of temperaturas and precipitation». Journal of Geophysical Research Atmospheres. DO5109, doi: 10. 1029/2005. JD 006290.

BRUNET INDIA, M. (2010): «Datos e indicadores para detectar y atribuir eventos al cambio climático: Los registros históricos del clima y su problemática». RDE (Realidad, Datos y Espacio. Revista Internacional de Estadística y Geografía. INEG. México). Año 1 nº 1, 19-29.

BROECKER, W. S. (1975): «Climate Change: Are We on the Brink of a Pronounced Global Warming?». Science (American Association for the Advancement of Science). New Serie Vol. 189 ( ${ }^{\circ}$ 4201, agosto), 460-463.

CALLE, A. et al. (2011): «Una revisión de las principales magnitudes relacionadas con la caracterización de los sensores usados en teledetección». Ponencia en XIV Congreso de la Asociación Española de Teledetección (Mieres del Camino, 2123 de sep., 2011): Bosques y cambio climático. Eds: Recondo, C. y Pendás, E., 473-476. En línea: http://156.35.47.3/docs/Teledetecci\%C3\%B3n\%20 Bosques\%20y\%20cambio\%20clim\%C3\%A1tico-ed_digital.pdf, Consultado el 15 de enero de 2012.

CASElles MiRAlles, V. et al. (1997): «La medida de la temperatura y de la emisividad desde satélites: Estado actual y perspectivas futuras». En Teledetección. Usos y aplicaciones. VI Reunión científica de la Asociación Española de Teledetección en 1995. Casanova, J.L et al. (Eds.). Secretariado de Publicaciones e Intercambio Científico Universidad de Valladolid. 487-492.

ChUvieco, E. (1993): «Teledetección, SIG y Cambio Global». V Coloquio de Geografía Cuantitativa (Zaragoza 1992). Ed. IFC-Dpto. de Geografía y Ordenación del Territorio. Universidad de Zaragoza, 33-55.

CHUVIECO, E. (2011): «Incendios forestales y Cambio Global». Ponencia invitada en XIV Congreso de la Asociación Española de Teledetección (Mieres del Camino, 21-23 de sep., 2011): Bosques y cambio climático. Eds: Recondo, C. y Pendás, E., (90 pp. en Power Point). En línea: http://156.35.47.3/, Consultado el 30 de enero de 2012.

DuQue, P. (2012): «Invertir en ciencia...por nuestros hijos». Diario El País. (10 de noviembre 2012), 39.

FONT, J. et al. (2011): «El proyecto SMOS». Ponencia invitada en XIV Congreso de la Asociación Española de Teledetección (Mieres del Camino, 21-23 de sep., 2011): Bosques y cambio climático. Eds: Recondo, C. y Pendás, E., (54 pp. en Power Point). En línea: http://156.35.47.3/, Consultado el 18 de enero de 2012. 
GALLO, P. et al. (1993): «The use of NOAA AVHRR data for assessment of the urban heat island effect». Journal of applied meteorology (American Meteorological Society). Vol 32 (n ${ }^{\circ}$ 5, mayo), 899-908.

GARCÍA-SOTO, C. (2002): «Navidad development in the southern Bay of Biscay: Climate Change and swoddy structure from remote sensing and in situ measurements». Journal of Geophysical Research. Vol. 107, 3118, doi: 1029/201 JC001012.

HegerL, G. C. et al. (2007): «Understanding and Attributing Climate Change». En Climate Change 2007: The Physical Science Basis. Contribution of Working Group I to the $4^{\circ}$ Assessment Report of the Intergovernmental Panel on Climate Change (Solomon, S. et al. Eds). Cambridge University Press. Cambridge (UK) and New York (USA).

JustiCE CH, O. et al. (1998): «The Moderate Resolution Imaging Spectoradiometer (MODIS): Land Remote Sensing for Global Change Research». IEEE Transactions on Geoscience and Remote Sensing. Vol. 36 (nº 4, julio), 1228-1249.

LAS HERAS, F. et al. (2010): «Puesta en marcha de una estación de seguimiento de satélites medioambientales para la monitorización y el estudio de los principales parámetros relacionados con el Cambio Climático». Medio Ambiente y Cambio Climático. Cuaderno Red de cátedras de Telefónica: "Las TIC aplicadas al medio ambiente. Los satélites y la observación del cambio climático”. Cátedra Telefónica de la Universidad de Oviedo. 12 pp. En línea: http://www. rcysostenibilidad.telefonica.com/blogs/documentoscatedras/files/2011/01/cuaderno-satelitesy-cambio-climatico -uniovi-v6.pdf, Consultado el 18 de agosto de 2011.

LOMBA FERRERAS, J. (2011): «Programa Nacional de Observación de la Tierra por Satélite». Ponencia invitada en XIV Congreso de la Asociación Española de Teledetección (Mieres del Camino, 21-23 de sep., 2011): Bosques y cambio climático. Eds: Recondo, C. y Pendás, E., (29 pp. en Power Point). En línea: http://156.35.47.3/, Consultado el 25 de febrero de 2012.

MARTíN-VIDE, J. (2008): «La nueva realidad del calentamiento global. Un decálogo del cambio climático». Scripta Nova. Revista Electrónica de Geografía y Ciencias Sociales. Barcelona, Universidad de Barcelona, 1 de agosto de 2008, vol. XII, no 27 (23) (nueva serie de “Geo-Crítica”. Cuadernos Críticos de Ga Humana). En línea: http://www.ub.edu/geocrit/sn/sn-270/sn-270-23.htm, Consultado el $01 / 12 / 2010$.

MilleR, CH. (2009): «The detection and Attribution of Climate Change» 25/06/2012, http/oar.noaa.gov/spotlite/archive/spot_ccdetect.html.

NicLós, R. et al. (2011): «Estimación de la temperatura del aire a nivel superficial a partir de imágenes de satélite». Ponencia en XIV Congreso de la Asociación Española de Teledetección (Mieres del Camino, 21-23 de sep., 2011): Bosques y cambio climático. Eds: Recondo, C. y Pendás, E., 449-452. En línea: http://156.35.47.3/docs/Teledetecci\%C3\%B3n\%20Bosques\%20y\%20cambio\%2 0clim\%C3\%A1tico-ed_digital.pdf . Consultado el 15 de enero de 2012.

OlIVIER, A. (2011): «The ESA Hearth Observation. Programmes: status and outlook». Ponencia invitada en XIV Congreso de la Asociación Española de Teledetección (Mieres del Camino, 21-23 de sep., 2011): Bosques y cambio climá- 
tico. Eds: Recondo, C. y Pendás, E., (76 pp. en Power Point). En línea: http://156.35.47.3/, Consultado el 25 de enero de 2012.

QUIRÓS HERNÁNDEZ, M. (2001): Teledetección y clima en Castilla y León: Distribución de las isotermas de las máximas. Valladolid. Ed. Universidad de Valladolid.

QUIRÓS HERNÁNDEZ, M. (2011): Tecnologías de la Información Geográfica (TIG). Cartografía, Fotointerpretación, Teledetección y SIG. Salamanca. Ediciones Universidad de Salamanca.

REDONDO, C. et al. (2011): «Estimación de la temperatura del aire a partir de las temperaturas de superficie obtenida con el sensor MODIS e información espacio-temporal. Aplicación en modelos de riesgo de incendios forestales en la Península Ibérica». Ponencia en XIV Congreso de la Asociación Española de Teledetección (Mieres del Camino, 21-23 de sep., 2011): Bosques y cambio climático. Eds: Recondo, C. y Pendás, E., 457-460. En línea: http://156.35.47.3/ docs/Teledetecci\%C3\%B3n\%20Bosques\%20y\%20cambio\%20clim\%C3\%A1tic o-ed_digital.pdf, Consultado el 15 de enero de 2012.

SACHS, J. D. (2012): «Un verano muy revelador». El País. (Suplemento Negocios, 12 de agosto 2012), p. 13.

SÁNCHEZ LORENZO, A et al. (2012): «Summer night-time temperature trends on de Iberian Peninsula and their connetion with large-scale atmospheric circulation patterns». International Journal of Climatology. Vol. 32 (9). 1326-1335.

SELLERS, P.J. et al. (1995): "Remote Sensing of the Land Surface for studies of Global Change». Remote Sensing of Enviroment (Ed. Elseiver). Vol 51 (Issue 1, enero), 3-26.

VILLA, G. (2011): «Plan Nacional de Teledetección. Estado actual y perspectivas futuras». Ponencia invitada en XIV Congreso de la Asociación Española de Teledetección (Mieres del Camino, 21-23 de sep., 2011): Bosques y cambio climático. Eds: Recondo, C. y Pendás, E., (133 pp. en Power Point). En línea: http://156.35.47.3/, Consultado el 15 de febrero de 2012.

WHITE, J. W et al. (2008): «Evaluation of NASA satellite -and assimilation modelderived long-term daily temperature data over the continental U.S.». En Agricultural and Forest Meteorology (Ed. Elsevier), n $148,1.574-1.584$.

ZEEBE, R. (2012a): «History of seawater carbonate chemistry, atmospheric $\mathrm{CO}_{2}$, and oceanic acidification». Annual Review of Earht and Planetary Sciences, vol. 40, (mayo 2012), 141-165. DOI: 10.1146/annurev-earth-042711-105521

ZEEBE, R. (2012b): «Climate Change and Ocean Adification» University of Hawai. Conferencia, junio 2012. En línea: http://vimeo.com/44771728, Consultado el 6 de noviembre de 2012. 